\title{
THE MOMENTUM-SPACE BOSONIZATION OF THE NAMBU-JONA-LASINIO MODEL WITH VECTOR AND AXIAL-VECTOR MESONS
}

\author{
Véronique Bernard, Ulf-G. Meißner \\ Centre de Recherches Nucléaires et Université Louis Pasteur de Strasbourg \\ Physique Théorique \\ BP 20Cr, 67037 Strasbourg Cedex 2, France \\ and \\ A.A. Osipov \\ Joint Institute for Nuclear Research, Laboratory of Nuclear Problems \\ 141980 Dubna, Moscow Region, Russia
}

\begin{abstract}
The momentum-space bosonization method is extended to the case of a Nambu-Jona-Lasinio type model with vector and axial-vector mesons. The method presented gives the possibility of deriving any meson vertex function to all orders in momenta and to the leading order in $1 / N_{c}$. Two-point functions, which describe one-particle transitions to the hadronic vacuum, and meson selfenergies are considered. We find new relations which generalize the well-known KSFR relation and both the first and the second Weinberg sum rules. These result from a consistent treatment of higher order terms in the momentum expansion.
\end{abstract}

CRN 93-57

December 1993 


\section{Introduction}

The Nambu-Jona-Lasinio (NJL) model in a version incorporating all essential QCD symmetries may be a reasonable low-energy approximation for QCD. In our previous paper [1] we suggested a systematic method for evaluating any mesonic N-point function in the bosonized NJL model. The main idea consists in the construction of special bosonic variables to be used for the description of the observable mesonic states. As a result, it is possible to extend the usual treatment of bosonized NJL models, which was formulated in papers [2] and developed in Refs. [3]-[6]. The standard approach is essentially oriented to the derivative expansion of the effective meson Lagrangian. Our method does not use this approximation. Therefore, one can expect it to be a powerful tool for exploration of the extended NJL model, which includes not only scalar and pseudoscalar fields but also heavier vector and axial-vector meson states.

One of the principal difficulties we come across on going over to higher energies that are typical of vector particles is the confinement property of QCD. The NJL model enables one to see dynamic symmetry breaking mechanism at work, which leads to meson formation from quark-antiquark pairs, but it does not forbid emission of constituent quarks (with mass $m$ ) into the continuum. The model has to deal with this phenomenon already at energies $p^{2} \sim 4 m^{2} \sim m_{\rho}^{2}$. In its formal part, our method does not require an a priori solution to the confinement problem. It only covers the general bosonization scheme for the model and mainly applies to the separation procedure of the collective degrees of freedom in theories with four-fermion interaction. For that aspect the confinement mechanism is not of relevance. Our goal here is to construct a formal scheme which involves the bosonization procedure and has all the advantages of the pure fermionic approach (Hartree-Fock plus Bethe-Salpeter approximation). The advantages of this approach are the explicit use of boson variables for describing the dynamics of collective excitations and the possibility of gaining full information on the momentum dependence of vertex functions.

The NJL model belongs to the set of nonrenormalizable theories. Hence, to define it completely as an effective model, a regularization scheme must be specified to deal with the quark-loop integrals in harmony with general symmetry requirements. As a result, an additional parameter $\Lambda$ appears, which characterizes the scale of the quark-antiquark forces responsible for the dynamic chiral symmetry breaking. From the meson mass spectrum it is known that $\Lambda \sim 1 \mathrm{GeV}$. Here, we will make use of the Pauli-Villars [7] regularization, which preserves gauge invariance. In this form it was used in Refs.[8]. 
For simplicity we consider the linear bosonized version of the extended NJL model with $U(2) \otimes U(2)$ symmetry which can be explicitly violated by the current quark masses. The extension to the case of $U(3) \otimes U(3)$ symmetry will be done elsewhere.

\section{Momentum-space bosonization of the extended NJL model}

Consider the extended $U(2) \otimes U(2)$ NJL Lagrangian with a local four-quark interaction

$$
\begin{gathered}
\mathcal{L}(q)=\bar{q}(i \not \partial-\widehat{m}) q+\frac{G_{S}}{2}\left[\left(\bar{q} \tau_{a} q\right)^{2}+\left(\bar{q} i \gamma_{5} \tau_{a} q\right)^{2}\right] \\
-\frac{G_{V}}{2}\left[\left(\bar{q} \gamma^{\mu} \tau_{a} q\right)^{2}+\left(\bar{q} \gamma_{5} \gamma^{\mu} \tau_{a} q\right)^{2}\right]
\end{gathered}
$$

where $\bar{q}=(\bar{u}, \bar{d})$ are coloured current quark fields with current mass $\widehat{m}=$ $\operatorname{diag}\left(\widehat{m}_{u}, \widehat{m}_{d}\right), \tau_{a}=\left(\tau_{0}, \tau_{i}\right), \tau_{0}=I, \tau_{i}(i=1,2,3)$ are the Pauli matrices of the flavour group $S U(2)_{f}$. The constants of the four-quark interactions are $G_{S}$ for the scalar and pseudoscalar cases, $G_{V}$ for the vector and the axial-vector cases. The current mass term explicitly violates the $U(2) \otimes U(2)$ chiral symmetry of the Lagrangian (2.1). In what follows, we shall only consider the isospin symmetric case $\widehat{m}_{u}=\widehat{m}_{d}=\widehat{m}$. Introducing boson fields in the standard way, the Lagrangian takes the form

$$
\begin{aligned}
\mathcal{L}(q, \bar{\sigma}, \tilde{\pi}, \tilde{v}, \tilde{a}) & =\bar{q}\left(i \not \partial-\widehat{m}+\bar{\sigma}+i \gamma_{5} \tilde{\pi}+\gamma^{\mu} \tilde{v}_{\mu}+\gamma_{5} \gamma^{\mu} \tilde{a}_{\mu}\right) q \\
& -\frac{\bar{\sigma}_{a}^{2}+\tilde{\pi}_{a}^{2}}{2 G_{S}}+\frac{\tilde{v}_{\mu a}^{2}+\tilde{a}_{\mu a}^{2}}{2 G_{V}} .
\end{aligned}
$$

Here $\bar{\sigma}=\bar{\sigma}_{a} \tau_{a}, \quad \tilde{\pi}=\tilde{\pi}_{a} \tau_{a}, \tilde{v}_{\mu}=\tilde{v}_{\mu a} \tau_{a}, \tilde{a}_{\mu}=\tilde{a}_{\mu a} \tau_{a}$. The vacuum expectation value of the scalar field $\bar{\sigma}_{0}$ turns out to be different from zero $\left(<\bar{\sigma}_{0}>\neq 0\right)$. To obtain the physical field $\tilde{\sigma}_{0}$ with $\left\langle\tilde{\sigma}_{0}\right\rangle=0$ one performs a field shift leading to a new quark mass $m$ to be identified with the mass of the constituent quarks

$$
\bar{\sigma}_{0}-\widehat{m}=\tilde{\sigma}_{0}-m, \quad \bar{\sigma}_{i}=\tilde{\sigma}_{i},
$$

where $m$ is determined from the gap equation (see Eq.(2.6) below).

Let us integrate out the quark fields in the generating functional associated with the Lagrangian (2.2). Evaluating the resulting quark determinant by a loop expansion one obtains

$$
\begin{aligned}
\mathcal{L}(\tilde{\sigma}, \tilde{\pi}, \tilde{v}, \tilde{a}) & =-i \operatorname{Tr} \ln \left[1+(i \not \partial-m)^{-1}\left(\tilde{\sigma}+i \gamma_{5} \tilde{\pi}+\gamma^{\mu} \tilde{v}_{\mu}+\gamma_{5} \gamma^{\mu} \tilde{a}_{\mu}\right)\right]_{\Lambda} \\
& -\frac{\bar{\sigma}_{a}^{2}+\tilde{\pi}_{a}^{2}}{2 G_{S}}+\frac{\tilde{v}_{\mu a}^{2}+\tilde{a}_{\mu a}^{2}}{2 G_{V}} .
\end{aligned}
$$


The index $\Lambda$ indicates that a regularization of the divergent loop integrals is introduced. We apply here the Pauli-Villars regularization [7], which preserves vector gauge invariance and at the same time might allow to reproduce the quark condensate for physical values of the current quark mass [6]. The Pauli-Villars cut-off $\Lambda$ is introduced by the following replacements

$$
\begin{gathered}
e^{-i m^{2} z} \rightarrow R(z)=e^{-i m^{2} z}\left[1-\left(1+i z \Lambda^{2}\right) e^{-i z \Lambda^{2}}\right], \\
m^{2} e^{-i m^{2} z} \rightarrow i R^{\prime}(z)=m^{2} R(z)-i z \Lambda^{4} e^{-i z\left(\Lambda^{2}+m^{2}\right)},
\end{gathered}
$$

where the minimal number of Pauli-Villars regulator has been introduced. In this case the expressions for some loop integrals $I_{i}$ (see formulae $(2.7),(2.11)$, and (2.24)) coincide with those obtained by the usual covariant cut-off scheme.

Consider the first terms of the expansion (2.4). From the requirement for the terms linear in $\tilde{\sigma}$ to vanish we get a modified gap equation

$$
m-\widehat{m}=8 m G_{P} I_{1}
$$

The integral $I_{1}$ is equal to

$$
I_{1}=i N_{c} \int^{\Lambda} \frac{d^{4} q}{(2 \pi)^{4}\left(q^{2}-m^{2}\right)}=\frac{3}{(4 \pi)^{2}}\left[\Lambda^{2}-m^{2} \ln \left(1+\frac{\Lambda^{2}}{m^{2}}\right)\right]
$$

where $N_{c}=3$ is the number of colours.

The terms quadratic in the boson fields lead to the amplitudes

$$
\begin{aligned}
\Pi^{\tilde{\pi} \tilde{\pi}}\left(p^{2}\right)= & {\left[8 I_{1}-G_{S}^{-1}+p^{2} g^{-2}\left(p^{2}\right)\right] \varphi_{\tilde{\pi}^{a}}^{+} \varphi_{\tilde{\pi}^{a}}^{-} } \\
\Pi^{\tilde{\sigma} \tilde{\sigma}}\left(p^{2}\right)= & {\left[8 I_{1}-G_{S}^{-1}+\left(p^{2}-4 m^{2}\right) g^{-2}\left(p^{2}\right)\right] \varphi_{\tilde{\sigma}^{a}}^{+} \varphi_{\tilde{\sigma}^{a}}^{-}, } \\
\Pi^{\tilde{v} \tilde{v}}\left(p^{2}\right)= & {\left[g^{\mu \nu} G_{V}^{-1}+4\left(p^{\mu} p^{\nu}-g^{\mu \nu} p^{2}\right) g_{V}^{-2}\left(p^{2}\right)\right] \varepsilon_{\mu}^{* \tilde{v}^{a}}(p) \varepsilon_{\nu}^{\tilde{v}^{a}}(p), } \\
\Pi^{\tilde{a} \tilde{a}}\left(p^{2}\right)= & {\left[g^{\mu \nu}\left(G_{V}^{-1}+4 m^{2} g^{-2}\left(p^{2}\right)\right)\right.} \\
& \left.+4\left(p^{\mu} p^{\nu}-g^{\mu \nu} p^{2}\right) g_{V}^{-2}\left(p^{2}\right)\right] \varepsilon_{\mu}^{* \tilde{a}^{a}}(p) \varepsilon_{\nu}^{\tilde{a}^{a}}(p), \\
\Pi^{\tilde{\pi} \tilde{a}}\left(p^{2}\right)= & 2 i m g^{-2}\left(p^{2}\right) p^{\mu} \varepsilon_{\mu}^{* \tilde{a}^{a}}(p) \varphi_{\tilde{\pi}^{a}}^{-}, \\
\Pi^{\tilde{a} \tilde{\pi}}\left(p^{2}\right)= & -2 i m g^{-2}\left(p^{2}\right) p^{\mu} \varepsilon_{\mu}^{\tilde{a}^{a}}(p) \varphi_{\tilde{\pi}^{a}}^{+} .
\end{aligned}
$$

Here $\varepsilon_{\mu}^{\tilde{v}^{a}}(p), \varepsilon_{\mu}^{\tilde{a}^{a}}(p)$ are the polarization vectors of the vector and axial-vector fields. We have introduced the symbols $\varphi_{\tilde{\pi}^{a}}^{-}=1$ and $\varphi_{\tilde{\sigma}^{a}}^{-}=1$ to explicitely 
show the pseudoscalar and scalar field contents of the pertinent two-point functions. The functions $g\left(p^{2}\right)$ and $g_{V}\left(p^{2}\right)$ are determined by the following integrals

$$
\begin{gathered}
g^{-2}\left(p^{2}\right)=4 I_{2}\left(p^{2}\right), \\
g_{V}^{-2}\left(p^{2}\right)=\frac{2}{3} J_{2}\left(p^{2}\right), \\
I_{2}\left(p^{2}\right)=\frac{3}{16 \pi^{2}} \int_{0}^{1} d y \int_{0}^{\infty} \frac{d z}{z} R(z) e^{i z \frac{p^{2}}{4}\left(1-y^{2}\right)}, \\
J_{2}\left(p^{2}\right)=\frac{9}{32 \pi^{2}} \int_{0}^{1} d y\left(1-y^{2}\right) \int_{0}^{\infty} \frac{d z}{z} R(z) e^{i z \frac{p^{2}}{4}\left(1-y^{2}\right) .}
\end{gathered}
$$

Let us diagonalize the quadratic form $(2.8 a)+(2.8 d)+(2.8 e)+(2.8 f)$ redefining the axial fields

$$
\begin{aligned}
\varepsilon_{\mu}^{\tilde{a}^{a}}(p) & \rightarrow \varepsilon_{\mu}^{\tilde{a}^{a}}(p)-i \beta\left(p^{2}\right) p_{\mu} \varphi_{\tilde{\pi}^{a}}^{-}, \\
\varepsilon_{\mu}^{* \tilde{a}^{a}}(p) & \rightarrow \varepsilon_{\mu}^{* \tilde{a}^{a}}(p)+i \beta\left(p^{2}\right) p_{\mu} \varphi_{\tilde{\pi}^{a}}^{+} .
\end{aligned}
$$

This determines the function $\beta\left(p^{2}\right)$,

$$
\beta\left(p^{2}\right)=\frac{8 m I_{2}\left(p^{2}\right)}{G_{V}^{-1}+16 m^{2} I_{2}\left(p^{2}\right)} .
$$

Consequently, one has no more mixing between pseudoscalar and axial-vector fields. The self-energy of the pseudoscalar field takes the form

$$
\Pi^{\tilde{\pi} \tilde{\pi}}\left(p^{2}\right)=\left[8 I_{1}-G_{S}^{-1}+p^{2} g^{-2}\left(p^{2}\right)\left(1-2 m \beta\left(p^{2}\right)\right)\right] \varphi_{\tilde{\pi}^{a}}^{+} \varphi_{\tilde{\pi}^{a}}^{-} .
$$

Now we can construct special boson variables that will describe the observed mesons. These field functions $\left(\pi_{a}, \sigma_{a}, v_{a}, a_{a}\right)$ correspond to bound quarkantiquark states and are derived via the following transformations

$$
\begin{aligned}
& \tilde{\pi}^{a}(p)=Z_{\pi}^{-1 / 2} g_{\pi}\left(p^{2}\right) \pi^{a}(p), \\
& \tilde{\sigma}^{a}(p)=Z_{\sigma}^{-1 / 2} g\left(p^{2}\right) \sigma^{a}(p), \\
& \tilde{v}^{a}(p)=\frac{1}{2} Z_{v}^{-1 / 2} g_{V}\left(p^{2}\right) v^{a}(p), \\
& \tilde{a}^{a}(p)=\frac{1}{2} Z_{a}^{-1 / 2} g_{V}\left(p^{2}\right) a^{a}(p),
\end{aligned}
$$


where

$$
g_{\pi}\left(p^{2}\right)=\frac{g\left(p^{2}\right)}{\sqrt{1-2 m \beta\left(p^{2}\right)}}=g\left(p^{2}\right) \sqrt{1+16 m^{2} G_{V} I_{2}\left(p^{2}\right)} .
$$

The new bosonic fields have the self-energies

$$
\begin{aligned}
\Pi_{a b}^{\pi, \sigma}\left(p^{2}\right) & =\delta_{a b} Z_{\pi, \sigma}^{-1}\left[p^{2}-m_{\pi, \sigma}^{2}\left(p^{2}\right)\right] \\
\Pi_{\mu \nu, a b}^{v, a}\left(p^{2}\right) & =\delta_{a b} Z_{v, a}^{-1}\left\{p_{\mu} p_{\nu}-g_{\mu \nu}\left[p^{2}-m_{v, a}^{2}\left(p^{2}\right)\right]\right\}
\end{aligned}
$$

The $p^{2}$-dependent masses are equal to

$$
\begin{aligned}
& m_{\pi}^{2}\left(p^{2}\right)=\left(G_{S}^{-1}-8 I_{1}\right) g_{\pi}^{2}\left(p^{2}\right), \\
& m_{\sigma}^{2}\left(p^{2}\right)=\left[1-2 m \beta\left(p^{2}\right)\right] m_{\pi}^{2}\left(p^{2}\right)+4 m^{2} \\
& m_{v}^{2}\left(p^{2}\right)=\frac{g_{V}^{2}\left(p^{2}\right)}{4 G_{V}}=\frac{3}{8 G_{V} J_{2}\left(p^{2}\right)}, \\
& m_{a}^{2}\left(p^{2}\right)=m_{v}^{2}\left(p^{2}\right)+6 m^{2} \frac{I_{2}\left(p^{2}\right)}{J_{2}\left(p^{2}\right)}
\end{aligned}
$$

The constants $Z_{\pi, \sigma, v, a}$ are determined by the requirement that the inverse meson field propagators $\Pi^{\pi, \sigma, v, a}\left(p^{2}\right)$ satisfy the normalization conditions

$$
\begin{aligned}
& \Pi^{\pi, \sigma}\left(p^{2}\right)=p^{2}-m_{\pi, \sigma}^{2}+\mathcal{O}\left(\left(p^{2}-m_{\pi, \sigma}^{2}\right)^{2}\right) \\
& \Pi_{\mu \nu}^{v, a}\left(p^{2}\right)=-g_{\mu \nu}\left[p^{2}-m_{v, a}^{2}+\mathcal{O}\left(\left(p^{2}-m_{v, a}^{2}\right)^{2}\right)\right]
\end{aligned}
$$

around the physical mass points $p^{2}=m_{\pi, \sigma, v, a}^{2}$, respectively. The conditions (2.20) lead to the values

$$
\begin{aligned}
& Z_{\pi}=1+\left.\frac{m_{\pi}^{2}\left[1-2 m \beta\left(m_{\pi}^{2}\right)\right]}{I_{2}\left(m_{\pi}^{2}\right)} \frac{\partial I_{2}\left(p^{2}\right)}{\partial p^{2}}\right|_{p^{2}=m_{\pi}^{2}}, \\
& Z_{\sigma}=1+\left.\frac{m_{\sigma}^{2}-4 m^{2}}{I_{2}\left(m_{\sigma}^{2}\right)} \frac{\partial I_{2}\left(p^{2}\right)}{\partial p^{2}}\right|_{p^{2}=m_{\sigma}^{2}}, \\
& Z_{v}=1+\left.\frac{m_{v}^{2}}{J_{2}\left(m_{v}^{2}\right)} \frac{\partial J_{2}\left(p^{2}\right)}{\partial p^{2}}\right|_{p^{2}=m_{v}^{2}}, \\
& Z_{a}=1+\left.\frac{m_{a}^{2}}{J_{2}\left(m_{a}^{2}\right)} \frac{\partial J_{2}\left(p^{2}\right)}{\partial p^{2}}\right|_{p^{2}=m_{a}^{2}}-\left.\frac{6 m^{2}}{J_{2}\left(m_{a}^{2}\right)} \frac{\partial I_{2}\left(p^{2}\right)}{\partial p^{2}}\right|_{p^{2}=m_{a}^{2}}
\end{aligned}
$$


Using the expressions (2.18), one can obtain the two-point Green functions $\Delta(p)$. For example, in the scalar and vector field case the relations

$$
\begin{aligned}
& \Pi_{a b}^{\sigma}\left(p^{2}\right) \Delta_{b c}^{\sigma}\left(p^{2}\right)=\delta_{a c} \\
& \Pi_{\mu \nu, a b}^{v}\left(p^{2}\right) \Delta_{b c}^{v, \nu \sigma}(p)=\delta_{a c} \delta_{\mu}^{\sigma} .
\end{aligned}
$$

give

$$
\begin{aligned}
\Delta_{a b}^{\sigma}\left(p^{2}\right) & =\frac{\delta_{a b} Z_{\sigma}}{p^{2}-m_{\sigma}^{2}\left(p^{2}\right)} \\
\Delta_{a b}^{v, \mu \nu}(p) & =\frac{\delta_{a b} Z_{v}}{m_{v}^{2}\left(p^{2}\right)} \frac{p^{\mu} p^{\nu}-g^{\mu \nu} m_{v}^{2}\left(p^{2}\right)}{p^{2}-m_{v}^{2}\left(p^{2}\right)} .
\end{aligned}
$$

The formal scheme developed here gives the possibility of evaluating any mesonic N-point Green function through the parameters of the model: $\Lambda, m, G_{S}, G_{V}$, and the one-loop integrals $I_{1}, I_{2}, J_{2}, I_{3}, \ldots I_{N}$. Two examples of these are

$$
\begin{aligned}
I_{2}\left(p^{2}\right) & =\int^{\Lambda} \frac{d^{4} q}{(2 \pi)^{4}} \frac{\left(-i N_{c}\right)}{\left(q^{2}-m^{2}\right)\left[(q+p)^{2}-m^{2}\right]} \\
I_{3}\left(p_{1}, p_{2}\right) & =\int^{\Lambda} \frac{d^{4} q}{(2 \pi)^{4}} \frac{\left(-i N_{c}\right)}{\left(q^{2}-m^{2}\right)\left[\left(q+p_{1}\right)^{2}-m^{2}\right]\left[\left(q+p_{2}\right)^{2}-m^{2}\right]} .
\end{aligned}
$$

This picture corresponds to the calculations in the framework of the pure fermionic NJL model where the Bethe-Salpeter equation sums an infinite class of fermion bubble diagrams.

\section{Scale-invariant relations and matching conditions}

The purpose of this section is to consider some general consequences of our approach and to compare them with the well-known current algebra results. We start with the form factor $f_{\pi}\left(p^{2}\right)$, which describes the week pion decay $\pi \rightarrow l \nu_{l}$ and can be expressed in the following form

$$
f_{\pi}\left(p^{2}\right)=\frac{m}{\sqrt{Z_{\pi}} g_{\pi}\left(p^{2}\right)}
$$

From here on, when omitting an argument of a running coupling constant or a running mass, we always assume that its value is taken on the mass-shell of the corresponding particle. The symbol of this particle will be used for that. For 
example, on the pion mass-shell $f_{\pi}\left(m_{\pi}^{2}\right)=f_{\pi}=93.3 \mathrm{MeV}$. Combining Eq.(2.19a) for the pion mass and the gap equation (2.6) with (3.1) one finds

$$
m_{\pi}^{2}\left(p^{2}\right) f_{\pi}^{2}\left(p^{2}\right)=\frac{\widehat{m} m}{Z_{\pi} G_{S}}=-\frac{2 \widehat{m}<\bar{q} q>}{Z_{\pi}\left(1-\frac{\widehat{m}}{m}\right)} .
$$

The right-hand side of this equality does not depend on $p^{2}$. This is an example of a scale-invariant relation that can be found in the model under consideration. It extends the well-known current-algebra result $m_{\pi}^{2} f_{\pi}^{2}=-2 \widehat{m}<\bar{q} q>$ derived by Gell-Mann, Oakes and Renner [9] that is exact at the lowest order of chiral expansion (in powers of external momenta $p^{2}$ and quark masses).

Another relation can be found in the sector of vector mesons. The form factor of the electromagnetic $\rho \rightarrow \gamma$ transition is equal to

$$
\frac{1}{f_{\rho}\left(p^{2}\right)}=\frac{2 g_{\rho}\left(p^{2}\right)}{3 \sqrt{Z_{\rho}}} J_{2}\left(p^{2}\right) \text {. }
$$

Using this formula and Eq.(2.19c) one can obtain the following scale-invariant result

$$
\frac{m_{\rho}^{2}\left(p^{2}\right)}{f_{\rho}^{2}\left(p^{2}\right)}=\frac{1}{4 Z_{\rho} G_{V}} .
$$

It is interesting to note here that the constant $G_{V}$ is related to the pion decay form factor too. This is a direct consequence of the $\pi-a_{1}$ mixing,

$$
\frac{f_{\pi}^{2}\left(p^{2}\right)}{m \beta\left(p^{2}\right)}=\frac{1}{2 Z_{\pi} G_{V}} \text {. }
$$

The comparison of the last two equations leads to a matching condition which relates physical quantities from different sectors of the model. In the particular case we deal with, properties of the $\rho$ meson and of the pion are related via

$$
m_{\rho}^{2}=a f_{\rho}^{2} f_{\pi}^{2}
$$

The constant $a$ in our case is equal to

$$
a=\frac{Z_{\pi}}{2 m Z_{\rho} \beta_{\pi}}
$$


For $a=2$ this is known as the KSFR relation [10]. The constant of the $\rho$ meson decay into two pions $f_{\rho \pi \pi}$ is usually included in the KSFR relation or eliminated from it as required by the universality condition $f_{\rho \pi \pi}=f_{\rho}$. Let us see to what extent the model behaviour of form factors agrees with this hypothesis. For that, we calculate the form factor $f_{\rho \pi \pi}\left(p^{2}\right)$,

$$
f_{\rho \pi \pi}\left(p^{2}\right)=\frac{g_{\rho}\left(p^{2}\right)}{\sqrt{Z_{\rho}}} F\left(p^{2}\right) .
$$

The function $F\left(p^{2}\right)$ represents the contribution of the $\rho \rightarrow \pi \pi$ triangular diagrams (including $\pi-a_{1}$ mixing effects) and has the form

$$
\begin{array}{r}
F\left(p^{2}\right)=\frac{1}{Z_{\pi}}\left\{1-\frac{m \beta_{\pi} p^{2}}{m_{\rho}^{2}\left(p^{2}\right)}+\frac{1-2 m \beta_{\pi}}{p^{2}-4 m_{\pi}^{2}}\left[\left(p^{2}-2 m_{\pi}^{2}\right)\left(\frac{I_{2}\left(p^{2}\right)}{I_{2}\left(m_{\pi}^{2}\right)}-1\right)\right.\right. \\
\left.\left.+2 m_{\pi}^{4} \frac{I_{3}\left(-p_{1}, p_{2}\right)}{I_{2}\left(m_{\pi}^{2}\right)}\right]\right\} .
\end{array}
$$

Here $p=p_{1}+p_{2}$, with $p_{1}, p_{2}$ the pion momenta. From this one easily deduces the following relation which holds off the $\rho$ mass shell

$$
\frac{f_{\rho \pi \pi}\left(p^{2}\right)}{f_{\rho}\left(p^{2}\right)}=\frac{F\left(p^{2}\right)}{Z_{\rho}} \text {. }
$$

In particular, at $p^{2}=0$ the equality

$$
I_{2}(0)-I_{2}\left(m_{\pi}^{2}\right)-\left.m_{\pi}^{2} I_{3}\left(-p_{1}, p_{2}\right)\right|_{p^{2}=0}=\left.2 m_{\pi}^{2} \frac{\partial I_{2}\left(p^{2}\right)}{\partial p^{2}}\right|_{p^{2}=m_{\pi}^{2}}
$$

leads to $F(0)=1$ and as a consequence one has $f_{\rho \pi \pi}(0)=f_{\rho}(0) / Z_{\rho}$.

Let us consider now the properties of the axial-vector meson $\left(a_{1}\right)$. For this purpose we have to calculate the axial-vector form factor $f_{a}\left(p^{2}\right)$ which describes the weak $a_{1} \rightarrow l \nu_{l}$ matrix element. The relevant calculations give

$$
\frac{1}{f_{a}\left(p^{2}\right)}=\frac{2 g_{\rho}\left(p^{2}\right)}{3 \sqrt{Z_{a}}} J_{2}\left(p^{2}\right)\left[1-2 m \beta\left(p^{2}\right)\right]=\sqrt{\frac{Z_{\rho}}{Z_{a}}} \frac{1-2 m \beta\left(p^{2}\right)}{f_{\rho}\left(p^{2}\right)}
$$

which leads to the result

$$
\frac{m_{a}^{2}\left(p^{2}\right)}{f_{a}^{2}\left(p^{2}\right)}=\frac{1-2 m \beta\left(p^{2}\right)}{4 Z_{a} G_{V}}
$$


where we used

$$
m_{a}^{2}\left(p^{2}\right)=\frac{m_{\rho}^{2}\left(p^{2}\right)}{1-2 m \beta\left(p^{2}\right)} .
$$

The relations (3.5) and (3.13) lead us to the another matching condition which relates the properties of the $a_{1}$ meson to pionic ones,

$$
m_{a}^{2}=b f_{a}^{2} f_{\pi}^{2}
$$

The constant $b$ is equal to

$$
b=\frac{Z_{\pi}\left(1-2 m \beta_{a}\right)}{2 m Z_{a} \beta_{\pi}} .
$$

From the relations (3.6) and (3.15) one can deduce a set of equivalent formulae. In particular, one of the direct consequences are the well-known Weinberg relations [11]. These are known to play a crucial role in the calculation of the electromagnetic $\pi^{+}-\pi^{0}$ mass difference in the chiral limit. An analogue of Weinberg's first sum rule is the equality

$$
Z_{a} \frac{m_{a}^{2}}{f_{a}^{2}}+Z_{\pi} \frac{\beta_{a}}{\beta_{\pi}} f_{\pi}^{2}=Z_{\rho} \frac{m_{\rho}^{2}}{f_{\rho}^{2}}
$$

The second sum rule is described by the relation

$$
Z_{a} \frac{m_{a}^{4}}{f_{a}^{2}}-Z_{\rho} \frac{m_{\rho}^{4}}{f_{\rho}^{2}}=\frac{m_{\rho}^{2}\left(m_{a}^{2}\right)-m_{\rho}^{2}}{4 G_{V}}
$$

The coefficients $Z, \beta$ and a non-zero right-hand side in (3.18) result from determining physical properties of particles at different values of $p^{2}$ corresponding to real values of their masses. In any approach where all physical characteristics of the mesons are fixed at a certain scale these relations take the standard form (in this case $\left.Z=1, \beta_{a}=\beta_{\pi}, m_{\rho}^{2}\left(m_{a}^{2}\right)=m_{\rho}^{2}\right)$. Off the mass shell (3.18) is replaced by the equality

$$
Z_{a} \frac{m_{a}^{4}\left(p^{2}\right)}{f_{a}^{2}\left(p^{2}\right)}=Z_{\rho} \frac{m_{\rho}^{4}\left(p^{2}\right)}{f_{\rho}^{2}\left(p^{2}\right)} .
$$




\section{Summary}

Let us briefly summarize our results. We have considered an extended NJL lagrangian with four quark interactions. It includes some of the salient features of QCD in the low-energy (long distance) regime. We have generalized the bosonization procedure of Ref.[1] to the case with vector and axial-vector mesons. This allows to calculate any mesonic $\mathrm{N}$-point function to all orders in momenta. In particular, we have considered some important relations connecting pion properties to ones of vector and axial-vector mesons. As specific examples we have shown that a generalized KSFR relation and generalized Weinberg sum rules are obtained from matching conditions relating the various sectors. These modified relations stem from a consistent treatment of the higher order terms in momenta. To lowest order one recovers the celebrated results $[10,11]$. Finally, we note that similar issues were studied in ref. [12]. There, however, all physical properties of mesons were fixed at $p^{2}=0$, which leads to substantial differences in the final results.

\section{REFERENCES}

[1] V.Bernard, A.A.Osipov and Ulf-G.Meißner, Phys.Lett. B285 (1992) 119.

[2] T.Eguchi, Phys.Rev. D14 (1976) 2755; K.Kikkawa, Progr.Theor.Phys. 56 (1976) 947.

[3] D.Ebert and M.K.Volkov, Yad.Fiz.36 (1982) 1265; Z.Phys. C16 (1983) 205.

[4] M.K.Volkov, Ann.Phys. 157 (1984) 282.

[5] A.Dhar and S.R.Wadia, Phys.Rev.Lett. 52 (1984) 959;

A.Dhar, R.Shankar and S.R.Wadia, Phys.Rev. D31 (1985) 3256.

[6] D.Ebert and H.Reinhardt, Nucl.Phys. B271 (1986) 188; Phys.Lett. B173 (1986) 453.

[7] W.Pauli and F.Villars, Rev.Mod.Phys. 21 (1949) 434.

[8] V.Bernard and D.Vautherin, Phys.Rev. D40 (1989) 1615;

C.Schüren, E.Ruiz Arriola and K.Goeke, Nucl.Phys. A547 (1992) 612.

[9] M.Gell-Mann, R.Oakes and B.Renner, Phys.Rev. 175 (1968) 2195.

[10] K.Kawarabayashi and M.Suzuki, Phys.Rev.Lett. 16 (1966) 255;

Riazuddin and Fayyazuddin, Phys.Rev. 147 (1966) 1071.

[11] S. Weinberg, Phys.Rev.Lett. 18 (1967) 507.

[12] J.Bijnens, E.de Rafael and H.Zheng, Preprint CERN-TH.6924/93 (1993). 